\title{
What is the most effective adhesive for attaching orthodontic bands?
}

\author{
What are the most effect adhesives to attach bands to teeth for fixed appliance \\ treatment?
}

Millett DT, Glenny AM, Mattick CR, Hickman J, Mandall NA.

Adhesives for fixed orthodontic bands. Cochrane Database of Systematic Reviews 2006; issue 3

Data sources The Cochrane Oral Health Group's Trials Register, the Cochrane Central Register of Controlled Trials (CENTRAL), Medline and Embase were used and a search of the internet also undertaken. There was no restriction with regard to publication status or language of publication.

Study selection Randomised controlled trials (RCT) and controlled clinical trials (CCT), including split-mouth studies, of adhesives used to attach orthodontic bands to molar teeth were selected.

Data extraction and synthesis All review authors were involved in study selection, validity assessment and data extraction without blinding to the authors, adhesives used or results obtained. All disagreements were resolved by discussion.

Results Five RCT and three CCT were identified as meeting the review's inclusion criteria. All the included trials were of split-mouth design. Four trials compared chemically cured zinc phosphate and chemically cured glass ionomer; three trials compared chemically cured glass ionomer cement with light-cured compomer; one trial compared chemically cured glass ionomer with a chemically cured glass phosphonate. Data analysis was often inappropriate within the studies that did meet the inclusion criteria.

Conclusions There is insufficient evidence to determine the most effective adhesive for attaching orthodontic bands to molar teeth in patients with full-arch fixed orthodontic appliances.
Address for correspondence: Luisa M Fernandez Mauleffinch, Cochrane Oral Health Group, MANDEC, School of Dentistry, University of Manchester, Higher Cambridge Street, Manchester M15 6FH, UK.

\section{Commentary}

A systematic review of the best adhesive for placing bands on teeth during fixed orthodontic treatment is indeed relevant and pertinent to most clinicians and researchers. In an age defined by time efficiency, the ability to retain bands for the entire duration of treatment would contribute a considerable efficiency and economy. The problem is that the quest for a perfect adhesive is not over and, unfortunately, this systematic review cannot provide any clinical bottom lines. It does most certainly highlight the lack of evidence and focused research, however, and thus provides a key to further objective work in the field.

A rigorous process and a critical eye for detail are demonstrated in the efforts of the reviewers. The searches are thorough, utilising all possible databases and including all RCT and CCT (and those with the split mouth technique), which provides a wide base to find suitable material for the review. The basic research question is simple and straightforward and the objectives are clear: which adhesive would best retain the band and for how long? Would it prevent decay around the bands?

The criteria for inclusion of studies in the review takes into account the types of participants, the interventions and the outcome measures. Data on the presence or absence of decalcification and decay is recorded, but the large disparity in the studies and the method of reporting this feature would obviously make it difficult for the reviewers to arrive at a clear conclusion. The method of review is thorough and takes into account almost all possible issues of bias and dissent.

Data synthesis is a challenge in a review with diverse study contents. The efforts to address the issues related to data synthesis are appreciable. Six main types of adhesives have been compared and, if possible, comparison was made within the groups between light- and chemical-cured adhesives.

The assessment of heterogeneity is thorough and precise. In view of these precise parameters, it is not surprising that only five RCT and three CCT out of a possible 24 trials met the requirements of the review. The inadequacy of evidence in literature today is highlighted by the qualitative comparison of various adhesives.

The reviewers suggest great caution in the interpretation of the results even in the broadest sense. During the past decade, twophase adhesives have gradually fallen from favour. Handling and applying these chemically cured adhesives is problematic, time-consuming and operator-sensitive. Mixing the two components introduces potentially critical defects such as surface porosity and air voids in the bulk material, because of the prolonged exposure to air and the inevitable entrapment of air bubbles, which adversely affect polymerisation because of the inhibition of the reaction by the oxygen in the atmospheric air. The principle of inhomogeneous polymerisation was introduced in orthodontics with the development of the no-mix adhesives, which were intended to minimise the mix- 
ing-induced defects and reduce the steps required for placement of the material. In these systems, a catalyst gradient is established from the primed enamel surface toward the brackets, by means of a diffusion process. Light-cured adhesives are the future, as succinctly suggested by Eliades recently in the American Journal of Orthodontics. ${ }^{1}$ Interpreting the results of the review here, the problems of the two-phase adhesives are clear: the reality is that most practitioners will abandon two-phase adhesives and light-cured composites will be the preferred option. The addition of various antimicrobial components to bonding is not new; the addition of fluoride-releasing components to adhesives to prevent or hinder microbial colonisation around bands and attachments has been already discussed in detail in the literature. ${ }^{2}$ With improvement in etching and single-phase adhesives, molar tube bonding is the future and a greater use of compomers and glass ionomers can be foreseen.

The absence of a clear clinical bottom line does not deride the importance of this review. The reality is that an evidence based review provides a clear perspective on all the issues related to adhesives and it certainly initiates a process of logical and critical inquiry in the mind of the clinician. And if this be so, then it just endorses the real objectives of evidence based science.

\section{Anmol Kalha}

Department of Orthodontics, Centre for Evidence Based Dentistry, College Of Dental Sciences, Davangere, Karnataka, India

1. Eliades T. Orthodontic materials research and applications. Part 1. Current status and projected future developments in bonding and adhesives. Am J Orthod Dentofacial Orthop 2006; 130:445-451.

2. Øgaard B, Larsson E, Henriksson T, Birkhed D, Bishara SE. Effects of combined application of antimicrobial and fluoride varnishes in orthodontic patients. Am J Orthod Dentofacial Orthop. 2001;120:28-35.

Evidence-Based Dentistry (2006) 7, 81-82. doi:10.1038/sj.ebd.6400449 